\title{
Lungenvolumenreduktion - H.-J. Schäfers chirurgisches Allheilmittel bei Emphysem?
}

\author{
Lung Volume Reduction - A Surgical Panacea?
}

Seit etwa 10 Jahren ist die chirurgische Volumenreduktion ein enthusiastisch und zum Teil auch kontrovers diskutiertes Thema in der Schnittfläche zwischen Thoraxchirurgie und Pneumologie. Der Beginn dieses neuen Therapieansatzes könnte aus der Retrospektive betrachtet einem Roman entstammen: Ein Chirurg stellt in (relativ) alter Zeit fest, dass mit der Entfernung von Lungengewebe die Dyspnoe bei Patienten mit Emphysem gebessert werden kann [1]. Es fehlte jedoch die physiologische Dokumentation der Ergebnisse. Ein weiterer, belesener und physiologisch geschulter Chirurg entdeckt die alte Beschreibung, setzt sie in ein physiologisches Konzept, und bringt diesen chirurgischen Ansatz in die Routine [2]. Somit besitzen nun Chirurgen einen sehr wirkungsvollen Ansatz für Patienten, bei denen konservative Therapiemöglichkeiten nur begrenzt helfen können $[3,4]$.

Der initiale Enthusiasmus über diese Erfolgsgeschichte einer chirurgischen Behandlung, die vor 20 Jahren nicht denkbar war, hat bereits begonnen sich zu legen. Bereits in den ersten Jahren der Erfahrung mit dieser Volumenreduktion wurde offensichtlich, dass es sich um einen belastenden Eingriff bei schwerkranken Patienten mit eingeschränkten physiologischen Reserven handelte. Dieser war nicht nur von erheblicher Morbidität, sondern auch Letalität begleitet [2,5]. Bald wurde auch offensichtlich, dass Patienten mit homogenem Emphysem zwar eine Verbesserung der Atemnot erfahren können, jedoch in Bezug auf objektive Parameter wenig Nutzen aus der chirurgischen Volumenreduktion ziehen [6]. Es stellte sich weiter heraus, dass Patienten mit einem basal betonten heterogenen Emphysem zwar eine drastische Verbesserung der Lungenfunktion früh nach dem Eingriff aufweisen, viele dieser Patienten jedoch bereits nach 12 Monaten funktionell nicht besser als vor dem Eingriff sind [6]. Bislang ist unklar, ob diese kurze „Halbwertzeit“ der Verbesserung der Lungenfunktion allein durch das spezifische Verteilungs- muster mit basaler Betonung der Bullae bedingt ist oder ob nicht vielmehr der bei diesen Patienten so häufige $\alpha_{1}$-Antitrypsinmangel die entscheidende Rolle im Rezidiv des Emphysem spielt.

Die gerade veröffentlichten Daten der National Emphysema Treatment Trials [7] werfen ein nüchternes, aber nicht vollkommen negatives Licht auf den außerordentlichen Enthusiasmus, mit dem viele Chirurgen weltweit dieses Werkzeug benutzten. Eine signifikante Verbesserung von objektiven messbaren Parametern und Überleben war bei Patienten nachweisbar, die eine deutliche Einschränkung von Lungenfunktion und körperlicher Leistungsfähigkeit aufwiesen und gleichzeitig ein heterogenes Emphysem besaßen mit apikaler Betonung. Die analysierte Nachuntersuchungszeit betrug 2 Jahre, und der nachweisbare Effekt erschien im gesamten Nachuntersuchungszeitraum konstant zu bleiben. Hiermit bestätigt diese große, multizentrisch angelegte Untersuchung die Berichte einzelner Gruppen, die eine signifikante Verbesserung von subjektiven und objektiven Parametern im Vergleich zur konservativen Behandlung einschließlich Rehabilitation bei einem Zeitraum von 2 bis 3 Jahren nachweisen konnten [2-4].

Zeigen uns diese Daten somit, dass die Lungenvolumenreduktion doch ein effektives Behandlungsverfahren ist, das zu subjektiver und objektiver Verbesserung führt und möglicherweise die Lebenserwartung verbessert? Die vorliegenden Daten können auch anders interpretiert werden. Bis auf die genannten Patienten mit apikal betontem Emphysem und schwerer Einschränkung der körperlichen Leistungsfähigkeit führt die Lungenvolumenreduktion nicht zu einer messbaren Verbesserung des Patienten. Dagegen ist sie von nennenswerter Morbidität und einer Letalität begleitet, die in erfahrenen Zentren mit exzellenter Infrastruktur in der Größenordnung von $5 \%$ liegt. In der positiv se-
Institutsangaben
Chirurgische Klinik, Abt. für Thorax- und Herz-Gefäßchirurgie, Homburg/Saar

Korrespondenzadresse

Prof. Dr. med. H.-J. Schäfers • Chirurgische Klinik, Abt. für Thorax- und Herz-Gefäßchirurgie • 66421 Homburg/Saar

Bibliografie

Pneumologie 2003; 57: 365-366 @ C Georg Thieme Verlag Stuttgart · New York · ISSN 0934-8387 
lektierten Subgruppe war die Volumenreduktion geringfügig besser in Bezug auf Kosteneffizienz als die medikamentöse Behandlung [8], bei allen anderen der - ohnehin im Rahmen der Studie hoch selektierten Patienten - führte die Volumenreduktion zu einer erheblichen Kostensteigerung ohne objektivierbaren Nutzen.

In Anbetracht der bei selektierten Patienten erzielten Verbesserung der Lungenfunktion nach Volumenreduktion erscheint es als durchaus akzeptabel, über den Einsatz eines solchen Prinzips im Zusammenhang anderer chirurgischer Verfahren nachzudenken. Die chirurgische Behandlung von Erkrankungen der Thoraxorgane hat sich ja in den letzten 50 Jahren zur Routine mit hohem Qualitätsniveau entwickelt, stößt jedoch immer wieder bei schwer fortgeschrittenen Erkrankungen der Lungen an physiologische Grenzen. Immer wieder werden Patienten, bei denen ein chirurgischer Eingriff - wie zum Beispiel die Resektion eines Bronchialkarzinoms - als potenziell lebensrettend gesehen werden muss, als funktionell „,inoperabel“ eingestuft. Gerade bei diesen Patienten ergeben sich dann wenig Alternativen, da auch die Strahlentherapie zu erheblichen funktionellen Konsequenzen durch die Begleitpneumonitis führen können. Die von Herrn Teschner verfasste Arbeit [9] stellt die interessante Hypothese auf, dass in bestimmten Konstellationen die Kombination aus onkologischer Lungenresektion und Lungenvolumenreduktion möglich ist und sogar zu sehr akzeptablen funktionellen Ergebnissen führt mit einer ausreichenden postoperativen Lebensqualität der betroffenen Patienten. Ist dieses ein Konzept, das so klar und erfolgreich ist, dass es in größerem Rahmen verfolgt werden sollte? - Letztendlich wirft die Arbeit mehr Fragen auf als sie beantwortet.

Die Volumenreduktion als alleiniger Eingriff an der Lunge ist in erfahrenen Zentren mit hervorragender Infrastruktur mit einer Letalität von $5 \%$ und einer ernst zu nehmenden Morbidität assoziiert. Die Zusammenfassung der 5 Kasuistiken lässt sicher keine Abschätzung zu, wie das Morbiditäts- und Letalitätsrisiko eines kombinierten Vorgehens zu bewerten ist, und ob möglicherweise das Letalitätsrisiko der Volumenreduktion den prognostischen Vorteil der onkologischen Resektion neutralisieren könnte.

Die Lungenvolumenreduktion ist bisher ein Eingriff, der nur einem kleinen, hochselektierten Anteil der Patienten mit Emphysem vorbehalten war. Trotz der bereits ausgeprägten Selektion zeigen die Daten des National Emphysema Treatment Trials, dass ein objektiver Vorteil durch die Behandlung nur bei einer Subgruppe dieser Patienten zu erzielen ist. Diese Beobachtungen legen den Schluss nahe, dass die Kombination aus Volumenreduktion und onkologischer Resektion bei nur einem verschwin- dend geringen Teil der betroffenen Patienten überhaupt mit Aussicht auf Erfolg infrage käme. Andererseits könnte der bei apikalem Emphysem nachgewiesene Überlebensvorteil auch in Kombination mit onkologischer Resektion die Langzeitergebnisse verbessern.

Welche chirurgischen Strategien sollten für diese Patienten entwickelt werden? - Die Antwort ist einfach, wenn ein superior betontes Raucheremphysem vorliegt, und der Oberlappen der Sitz des Bronchialkarzinoms ist. Liegt der Primärtumor dagegen in noch adäquat erhaltenem, basalem Lungengewebe, wird eine Resektion mit ausreichender onkologischer Konsequenz, d.h. Lobektomie, in der Regel funktionell nicht möglich sein. Der Sitz eines Tumors im emphysematösen Oberlappen wirft allerdings auch die Frage auf, ob unter diesen Bedingungen nicht allein schon die onkologisch orientierte Lobektomie einen eigenen volumenreduzierenden Effekt haben würde, ohne dass zusätzliche Maßnahmen an der Lunge erforderlich wären.

Es ist zunehmend wichtiger, dass sich der Thoraxchirurg mit der Physiologie der Lunge intensiv auseinandersetzt und versucht, diese in seinen therapeutischen Strategien zu berücksichtigen. Genauso wichtig ist es jedoch, standardisierbare Konzepte zu entwickeln und diese auf den Prüfstand kontrollierter und prospektiver Studien zu stellen.

\section{Literatur}

${ }^{1}$ Brantigan O, Mueller E. Surgical treatment of pulmonary emphysema. Am Surg 1957; 23: 789-804

${ }^{2}$ Cooper J, Trulock E, Triantafillou A et al. Bilateral pneumonectomy (volume reduction) for chronic obstructive pulmonary disease. J Thoracic Cardiovasc Surg 1995; 109: 106-116

${ }^{3}$ Gaissert H, Trulock E, Cooper J et al. Comparison of early functional results after volume reduction or lung transplantation for chronic obstructive pulmonary desease. J Thorac Cardiovasc Surg 1996; 111: 296-306

${ }^{4}$ Wilkens H, Demertzis S, König J et al. Lung volume reduction surgery versus conservative treatment in severe emphysema. Eur Respir J 2000; 16: 1043 - 1049

${ }^{5}$ National Emphysema Treatment Trial Research Group. Patients at high risk of death after lung-volume-reduction surgery. N Engl J Med 2001; 345: 1075-1083

${ }^{6}$ Fujimoto T, Teschler H, Hillejan L et al. Long-term result of lung volume reduction surgery. Eur J Cardiothorac Surg 2002; 21: 483-488

${ }^{7}$ Fishman A, Martinez F, Naunheim K et al. A randomized trial comparing lung-volume-reduction surgery with medical therapy for severe emphysema. N Eng J Med 2003; 348: 2059-2073

8 Ramsey SD, Berry K, Etzioni R et al. Cost effectiveness of lung-volumereduction surgery for patients with severe emphysema. N Engl J Med 2003; 348: 2092-2102

${ }^{9}$ Teschner M, Starp F, Lüllig H. Die Resektion von (NSCLC-)Bronchialkarzinomen mit simultaner Lungenvolumenreduktion bei terminalem Lungenemphysem. Pneumologie 2003; 57: $367-372$ 